\title{
FINITE DIFFERENCE METHOD FOR THE FRACTIONAL ORDER PSEUDO TELEGRAPH INTEGRO-DIFFERENTIAL EQUATION
}

\author{
Mahmut Modanli ${ }^{1}$, Fatih Ozbag ${ }^{1}$, Ali Akgül ${ }^{2 *}$ \\ ${ }^{1}$ Art and Science Faculty, Harran University \\ Sanliurfa, Turkey \\ ${ }^{2}$ Art and Science Faculty, Siirt University \\ Siirt, Turkey \\ mmodanli@harran.edu.tr,fozbag@harran.edu.tr,aliakgul00727@gmail.com
}

Received: 26 May 2021; Accepted: 12 September 2021

\begin{abstract}
The main goal of this paper is to investigate the numerical solution of the fractional order pseudo telegraph integro-differential equation. We establish the first order finite difference scheme. Then for the stability analysis of the constructed difference scheme, we give theoretical statements and prove them. We also support our theoretical statements by performing numerical experiments for some fractions of order $\alpha$.
\end{abstract}

MSC 2010: 65M06, 26A33

Keywords: pseudo telegraph equation, integro-differential equation, finite difference scheme, stability analysis

\section{Introduction}

Fractional calculus has become a valuable tool in mathematical modeling recently [1-9]. Although there are some different generalizations of the differential operator in the literature, Riemann-Liouville and Caputo fractional derivatives are the most commonly used definitions. The former type of fractional derivative is closely related to the latter type of fractional derivative. The definition of the Riemann-Liouville fractional derivative is

$$
D_{\xi}^{\alpha} u(\xi, v)=\frac{1}{\Gamma(n-\alpha)} \frac{\partial^{n}}{\partial \xi^{n}} \int_{0}^{\xi} \frac{1}{(\xi-s)^{\alpha-n+1}} u(s, v) d s,(n-1<\alpha \leq n) .
$$

The Caputo fractional derivative $D_{\xi}^{\alpha} u(\xi, v)$ of order $\alpha$ with respect to time is defined as:

$$
D_{\xi}^{\alpha} u(\xi, v)=\frac{1}{\Gamma(n-\alpha)} \int_{0}^{\xi} \frac{1}{(\xi-s)^{\alpha-n+1}} \frac{\partial^{n} u(s, v)}{\partial s^{n}} d s,(n-1<\alpha \leq n) .
$$


We consider both the Riemann-Liouville and Caputo type fractional order of pseudo telegraph integro-differential equation. An arbitrary function does not have to be continuous at the origin or differentiable to use the Riemann-Liouville fractional derivative. On the other side, one of the major advantages of the Caputo fractional derivative is that it allows for the inclusion of traditional initial and boundary conditions in the formulation of the problem. When dealing with real-world situations, the Caputo fractional derivative additionally allows the application of the initial and boundary conditions. When modeling real-world problems, the Caputo derivative is the best fractional operator to use.

Several numerical and analytical solution techniques are applied to fractional order telegraph equations [10-16]. Pseudo types of this equation contain a mixed partial derivative with respect to variables of time and space. In [17], the pseudo partial differential equation is used to model signal propagation along a neuristor which is a one-dimensional channel through which signals can flow and which is utilized to generate all digital logic functions. Also, the line for transmitting active pulses is extended to an active surface and modeled by pseudo equations. Therefore, these types of equations have a significant role in applied science, and it is important to obtain exact and approximate numerical solutions for either integer or non-integer order. In the literature there are several methods studied to solve these equations both analytically and numerically [18-25]. For solving the time-fractional Burger-Huxley equation inside the Caputo type fractional derivative, a simple and powerful numerical technique was provided [26]. In [27], to get solutions to the time fractional Advection-Diffusion equation, a powerful technique was devised. The fractional Boussinesq-like equation with the $\beta$ derivative, which explains the propagation of tiny amplitude long capillary-gravity waves on the surface of shallow water, was used in [28]. They proposed new solutions to the fractional-order Korteweg-de Vries problem by combining the benefits of fictitious time integration with group preservation methods [29]. In recent years, many studies have been done on the finite difference method [30-32].

We consider:

$$
\left\{\begin{array}{l}
u_{\xi \xi}(\xi, v)+u_{\xi}(\xi, v)+u(\xi, v)+\int_{0}^{\xi} \gamma(s) D_{s}^{\alpha} u(s, v) d s=u_{\xi v v}+u_{v v}(\xi, v)+f(\xi, v), \\
u(0, v)=u_{\xi}(0, v)=0, \quad 0<v<L \\
u(\xi, 0)=u(\xi, L)=0, \quad 0<\xi<T \\
0<\alpha<1
\end{array}\right.
$$

where $\gamma(\xi)$ is continuous on $0<\xi \leq T$ with constraint

$$
|\gamma(s)| \leq \frac{K}{s^{1-\alpha}}
$$


In the literature, there is no study about the approximate solution of this problem. For this reason, it is important to investigate the exact and approximate solutions of this problem. Obtaining difference schemes and making stability estimations for this problem make this study different.

The considered telegraph equation has an integral component, making it an integrodifferential equation with fractional derivative. Due to the difficulty of solving these equations analytically, an efficient approximation solution is necessary. This study presents a numerical solution of the problem (3). In order to calculate approximate numerical solutions, we begin with constructing the first order finite difference scheme. Then for the stability analysis of the constructed difference scheme, in the next section we give theoretical statements and prove them. We also support our theoretical statements by performing numerical experiments for some fractions of order $\alpha$. Error analysis for the numerical solutions are presented.

\section{Matrix stability for finite difference method}

We assume that $h=\frac{L}{M}$ for x-axis and $\tau=\frac{T}{N}$ for t-axis grid mess. Thus, we get

$$
v_{n}=n h, n=1,2, \ldots, M, \quad \xi_{k}=k \tau, k=1,2, \ldots, N
$$

For the fractional pseudo telegraph integro-differential equation (3), we establish the difference schemes by the finite difference method as:

$$
\left\{\begin{array}{l}
\frac{u_{n}^{k+1}-2 u_{n}^{k}+u_{n}^{k-1}}{\tau^{2}}+\frac{u_{n}^{k+1}-u_{n}^{k}}{\tau}+u_{n}^{k+1}+\sum_{l=1}^{k-1} \sum_{m=1}^{l} \frac{\Gamma(l-m+1-\alpha)\left(u_{n}^{m+1}-u_{n}^{m}\right)}{\Gamma(1-\alpha)(l-m) ! l^{1-\alpha}} \\
=\frac{1}{\tau}\left(\frac{u_{n+1}^{k+1}-2 u_{n}^{k+1}+u_{n-1}^{k+1}}{h^{2}}-\frac{u_{n+1}^{k}-2 u_{n}^{k}+u_{n-1}^{k}}{h^{2}}\right)+\frac{u_{n+1}^{k+1}-2 u_{n}^{k+1}+u_{n-1}^{k+1}}{h^{2}}+f_{n}^{k} \\
u_{n}^{0}=\varphi\left(v_{n}\right), \quad \frac{u_{n}^{1}-u_{n}^{0}}{\tau}=\psi\left(v_{n}\right) .
\end{array}\right.
$$


Then, we get

$$
\left\{\begin{array}{l}
{\left[\left(-\frac{1}{\tau h^{2}}-\frac{1}{h^{2}}\right) u_{n-1}^{k+1}+\left(\frac{1}{\tau^{2}}+\frac{1}{\tau}+1+\frac{2}{\tau h^{2}}+\frac{2}{h^{2}}\right) u_{n}^{k+1}+\left(-\frac{1}{\tau h^{2}}-\frac{1}{h^{2}}\right) u_{n+1}^{k+1}\right]} \\
+\left[\left(\frac{1}{\tau h^{2}}\right) u_{n-1}^{k}+\left(-\frac{2}{\tau^{2}}-\frac{1}{\tau}-\frac{2}{\tau h^{2}}\right) u_{n}^{k}+\left(\frac{1}{\tau h^{2}}\right) u_{n+1}^{k}\right]+\left[\left(\frac{1}{\tau^{2}}\right) u_{n}^{k-1}\right] \\
=f_{n}^{k}-\sum_{l=1}^{k-1} \sum_{m=1}^{l} \frac{\Gamma(l-m+1-\alpha)\left(u_{n}^{m+1}-u_{n}^{m}\right)}{\Gamma(1-\alpha)(l-m) ! l^{1-\alpha}}, \\
u_{n}^{0}=\varphi\left(v_{n}\right), \quad \frac{u_{n}^{1}-u_{n}^{0}}{\tau}=\psi\left(v_{n}\right), 1 \leq n \leq M, \\
u_{0}^{k}=u_{M}^{k}=0,0 \leq k \leq N .
\end{array}\right.
$$

Then, we obtain

$$
\left\{\begin{array}{l}
u^{1}=u^{0}+\tau \psi \\
A u^{k+1}=B u^{k}+C u^{k-1}+\varphi_{n}^{k}-\sum_{l=1}^{k-1} \sum_{m=1}^{l} \frac{\Gamma(l-m+1-\alpha)\left(u^{m+1}-u^{m}\right)}{\Gamma(1-\alpha)(l-m) ! l^{1-\alpha}} \\
u_{0}^{k}=u_{M}^{k}=0,0 \leq k \leq N
\end{array}\right.
$$

where $\varphi_{n}^{k}=\left[\varphi_{0}^{k}, \varphi_{1}^{k}, \ldots, \varphi_{M}^{k}\right], \varphi_{n}^{0}=\varphi\left(v_{n}\right), \varphi_{n}^{k}=f_{n}^{k}=f\left(\xi_{k}, v_{n}\right), 1 \leq n \leq M, 1 \leq k \leq$ $N$ and $u^{k}=\left[u_{0}^{k}, u_{1}^{k}, \ldots, u_{N}^{k}\right]^{T}$. Where $A$ and $B$ are symmetric tridiagonal matrices and $C$ is a diagonal matrix.

$$
A=\left[\begin{array}{ccccccc}
a & b & 0 & \cdots & 0 & 0 & 0 \\
b & a & b & \cdots & 0 & 0 & 0 \\
\vdots & \vdots & \vdots & \ddots & \vdots & \vdots & \vdots \\
0 & 0 & 0 & \cdots & b & a & b \\
0 & 0 & 0 & \cdots & 0 & b & a
\end{array}\right]_{(N-1) \times(N-1)}
$$

where $a=\frac{1}{\tau^{2}}+\frac{1}{\tau}+1+\frac{2}{\tau h^{2}}+\frac{2}{h^{2}}, b=-\frac{1}{\tau h^{2}}-\frac{1}{h^{2}}$,

$$
B=\left[\begin{array}{ccccccc}
k & p & 0 & \cdots & 0 & 0 & 0 \\
p & k & p & \cdots & 0 & 0 & 0 \\
\vdots & \vdots & \vdots & \ddots & \vdots & \vdots & \vdots \\
0 & 0 & 0 & \cdots & p & k & p \\
0 & 0 & 0 & \cdots & 0 & p & k
\end{array}\right]_{(N-1) \times(N-1)}
$$


where $k=\frac{2}{\tau^{2}}+\frac{1}{\tau}+\frac{2}{\tau h^{2}}, \quad p=-\frac{1}{\tau h^{2}}$,

$$
C=\left[\begin{array}{ccccccc}
c & 0 & 0 & \cdots & 0 & 0 & 0 \\
0 & c & 0 & \cdots & 0 & 0 & 0 \\
\vdots & \vdots & \vdots & \ddots & \vdots & \vdots & \vdots \\
0 & 0 & 0 & \cdots & 0 & c & 0 \\
0 & 0 & 0 & \cdots & 0 & 0 & c
\end{array}\right]_{(N-1) \times(N-1)}
$$

where $c=-\frac{1}{\tau^{2}}$.

We express $\|A\|=\|A\|_{\infty}=\max _{1 \leq j \leq N-1} \sum_{j=1}^{N-1}\left|a_{j m}\right|$ where $A=\left[a_{j m}\right]_{(N-1) \times(N-1)}$.

Lemma 1 Let $d=\sum_{l=1}^{k-1} \sum_{m=1}^{l} \frac{\Gamma(l-m+1-\alpha)}{\Gamma(1-\alpha)(l-m) ! l^{1-\alpha}}$. If $\frac{2}{\tau^{2}}+\frac{1}{\tau}+\frac{2}{\tau h^{2}}-d>0$ and $\frac{1}{\tau^{2}}+$ $\frac{4}{\tau h^{2}}-\frac{2}{h^{2}}-1-d<0$, then $\left\|A^{-1} B\right\| \leq 1$.

ProOF Let us assume $\frac{2}{\tau^{2}}+\frac{1}{\tau}+\frac{2}{\tau h^{2}}-d>0$ and $\frac{1}{\tau^{2}}+\frac{4}{\tau h^{2}}-\frac{2}{h^{2}}-1-d<0$, then

$$
\begin{aligned}
\left\|A^{-1} B\right\| \leq\left\|A^{-1}\right\|\|B\| & \leq \frac{1}{\min _{1 \leq j \leq N-1}\left\{\left|a_{j j}\right|-\sum_{m \neq j, m=1}^{N-1}\left|a_{j m}\right|\right\}}\|B\| \\
& \leq \frac{\left|\frac{2}{\tau^{2}}+\frac{1}{\tau}+\frac{2}{\tau h^{2}}-d\right|+\left|\frac{1}{\tau h^{2}}\right|+\left|\frac{1}{\tau h^{2}}\right|}{\left|\frac{1}{\tau^{2}}+\frac{1}{\tau}+1+\frac{2}{\tau h^{2}}+\frac{2}{h^{2}}\right|-\left|-\frac{1}{\tau h^{2}}\right|-\left|-\frac{1}{\tau h^{2}}\right|} \\
& =\frac{\frac{2}{\tau^{2}}+\frac{1}{\tau}+\frac{2}{\tau h^{2}}-d+\frac{2}{\tau h^{2}}}{\frac{1}{\tau^{2}}+\frac{1}{\tau}+1+\frac{2}{\tau h^{2}}+\frac{2}{h^{2}}-\frac{2}{\tau h^{2}}}=\frac{\frac{2}{\tau^{2}}+\frac{1}{\tau}+\frac{4}{\tau h^{2}}-d}{\frac{1}{\tau^{2}}+\frac{1}{\tau}+1+\frac{2}{h^{2}}} \\
& \leq 1
\end{aligned}
$$

since $\frac{1}{\tau^{2}}+\frac{4}{\tau h^{2}}-\frac{2}{h^{2}}-1<d$.

Lemma 2 Let $d=\sum_{l=1}^{k-1} \sum_{m=1}^{l} \frac{\Gamma(l-m+1-\alpha)}{\Gamma(1-\alpha)(l-m) ! l^{1-\alpha}}$. If $\frac{2}{\tau^{2}}+\frac{1}{\tau}+\frac{2}{\tau h^{2}}-d>0$, then $\left\|A^{-1} C\right\| \leq 1$. 
PROOF

$$
\begin{aligned}
\left\|A^{-1} C\right\| \leq\left\|A^{-1}\right\|\|C\| & \leq \frac{1}{\min _{1 \leq j \leq N-1}\left\{\left|a_{j j}\right|-\sum_{m \neq j, m=1}^{N-1}\left|a_{j m}\right|\right\}}\|C\| \\
& \leq \frac{\frac{1}{\tau^{2}}}{\frac{1}{\tau^{2}}+\frac{1}{\tau}+1+\frac{2}{h^{2}}} \\
& \leq 1 .
\end{aligned}
$$

Theorem 1 If $\frac{2}{\tau^{2}}+\frac{1}{\tau}+\frac{2}{\tau h^{2}}-d>0$ and $\frac{1}{\tau^{2}}+\frac{4}{\tau h^{2}}-\frac{2}{h^{2}}-1-d<0$, then the equation (6) is stable.

PROOF Utilizing a similar procedure in [10], and implementing Lemma 1 and 2, the proof of the theorem is completed.

\section{Computational examples}

\subsection{Example 1}

We consider

$$
\left\{\begin{array}{l}
u \xi \xi(\xi, v)+u_{\xi}(\xi, v)+u(\xi, v)+\int_{0}^{\xi} \gamma(s) D_{s}^{\alpha} u(s, v) d s=u_{\xi v v}(\xi, v)+u_{v v}(\xi, v)+f(\xi, v), \\
f(\xi, v)=\left(2+2 \xi+\left(1+\pi^{2}\right) \xi^{2}+2 \pi^{2} \xi+\frac{\xi^{2}}{\Gamma(3-\alpha)}\right) \sin (\pi v), 0<v<1,0<\xi<1, \\
u(0, v)=u_{\xi}(0, v)=0, \quad 0 \leq v \leq 1 \\
u(\xi, 0)=u(\xi, 1)=0, \quad 0 \leq \xi \leq 1 \\
0<\alpha \leq 1
\end{array}\right.
$$

The exact solution is given as $u(\xi, v)=\xi^{2} \sin (\pi v)$.

To solve this problem numerically, the established first order difference scheme is as follows: 


$$
\left\{\begin{array}{l}
\frac{u_{n}^{k+1}-2 u_{n}^{k}+u_{n}^{k-1}}{\tau^{2}}+\frac{u_{n}^{k+1}-u_{n}^{k}}{\tau}+u_{n}^{k+1}+\sum_{l=1}^{k-1} \sum_{m=1}^{l} \frac{\Gamma(l-m+1-\alpha)\left(u_{n}^{m+1}-u_{n}^{m}\right)}{\Gamma(1-\alpha)(l-m) ! l^{1-\alpha}} \\
=\frac{1}{\tau}\left(\frac{u_{n+1}^{k+1}-2 u_{n}^{k+1}+u_{n-1}^{k+1}}{h^{2}}-\frac{u_{n+1}^{k}-2 u_{n}^{k}+u_{n-1}^{k}}{h^{2}}\right)+\frac{u_{n+1}^{k+1}-2 u_{n}^{k+1}+u_{n-1}^{k+1}}{h^{2}}+f_{n}^{k} \\
v_{n}=n h, \xi_{k}=k \tau, 1 \leq k \leq N-1, \quad 1 \leq n \leq M-1, \\
u_{n}^{0}=0, \quad \frac{u_{n}^{1}-u_{n}^{0}}{\tau}=0, \quad 0 \leq n \leq M, \\
u_{0}^{k}=u_{M}^{k}=0,0 \leq k \leq N .
\end{array}\right.
$$

Then, writing the system in the matrix form, we have

$$
A u_{n+1}+B u_{n}+C u_{n-1}=D \varphi_{n}
$$

where $A, B$ and $C$ are $(N+1) \times(N+1)$ square matrices and $D$ is identity matrix.

To solve this resulting difference equation, the modified Gauss elimination method is applied. The derivation of the Gaussian elimination method was given in [11]. In Table 1, the approximate solutions for $\mathrm{N}$ and $\mathrm{M}$ are calculated for each $\alpha$ with $N^{2}=M$ (or $h=\tau^{2}$ ). The maximum norm error of the approximate solution is calculated by

$$
\varepsilon=\max _{\substack{1 \leq k \leq N \\ 1 \leq n \leq M}}\left|u(\xi, v)-u\left(\xi_{k}, v_{n}\right)\right|
$$

where $u(\xi, v)$ and $u\left(\xi_{k}, v_{n}\right)$ are exact and approximate solutions respectively. Then, we give the error plot of the difference scheme (10) in Figure 1 while changing time.

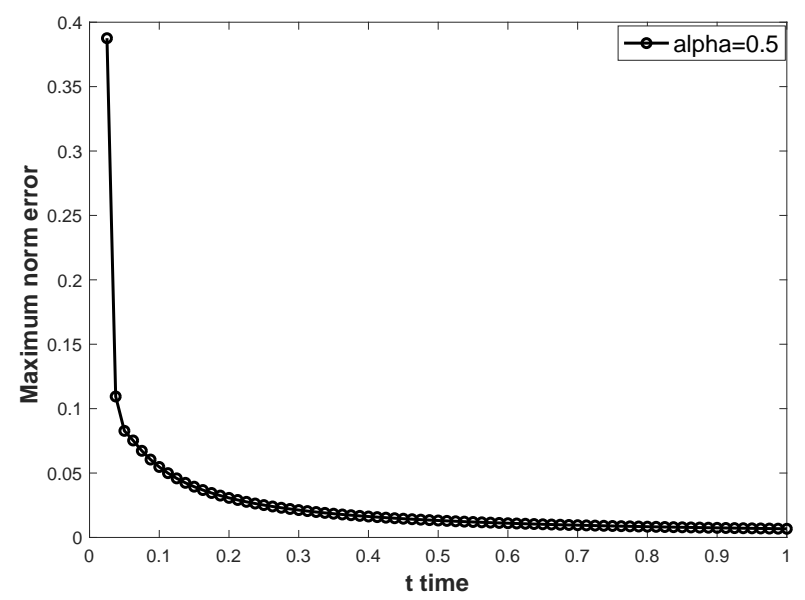

Fig. 1. Result of maximum norm errors of the difference scheme (10) for $\alpha=0.5$. As we vary the time values, it can be observed that errors keep decreasing 
To make the error computations more obvious, we present the error table for various values of $\alpha$.

Table 1. Error calculations

\begin{tabular}{|l|l|l|l|l|l|}
\hline \multicolumn{5}{|l|}{$\tau=\frac{1}{N}, h=\frac{1}{M}, h=\tau^{2}$} \\
\hline The example (9). \\
\hline $\mathrm{N}, \mathrm{M}$ & $\begin{array}{l}\mathrm{N}=20, \\
\mathrm{M}=20^{2}\end{array}$ & $\begin{array}{l}\mathrm{N}=40, \\
\mathrm{M}=40^{2}\end{array}$ & $\begin{array}{l}\mathrm{N}=80, \\
\mathrm{M}=80^{2}\end{array}$ & $\begin{array}{l}\mathrm{N}=100, \\
\mathrm{M}=100^{2}\end{array}$ & $\begin{array}{l}\mathrm{N}=150, \\
\mathrm{M}=150^{2}\end{array}$ \\
\hline$\alpha=0.01$ & 0.0244 & 0.0128 & 0.0066 & 0.0053 & 0.0035 \\
\hline$\alpha=0.5$ & 0.0251 & 0.0132 & 0.0067 & 0.0054 & 0.0036 \\
\hline$\alpha=0.99$ & 0.0263 & 0.0137 & 0.0070 & 0.0056 & 0.0038 \\
\hline
\end{tabular}

The accuracy of the developed scheme is confirmed by Table 1, which shows that as the grid points increase, the maximum norm error decreases. We then exhibit precise and numerical solutions for each $\alpha$ to demonstrate how similar the solutions are.

The exact solution and the numerical solution obtained for $\alpha=0.01$ with the step sizes $h=\frac{1}{6400}$ and $\tau=\frac{1}{80}$ are shown in Figure 2 .
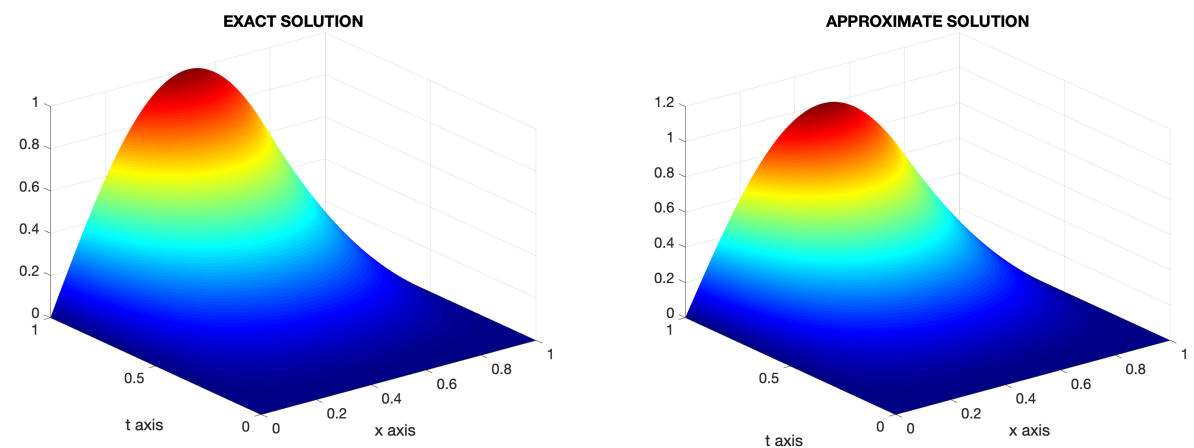

Fig. 2. Left: exact solution. Right: numerical simulation of the problem (9) for $\mathrm{N}=80, \mathrm{M}=6400$ and $\alpha=0.01$

For $\alpha=0.5$ with the same step sizes as in the previous figure, the exact and numerical solutions obtained for the problem (9) are presented in Figure 3. 

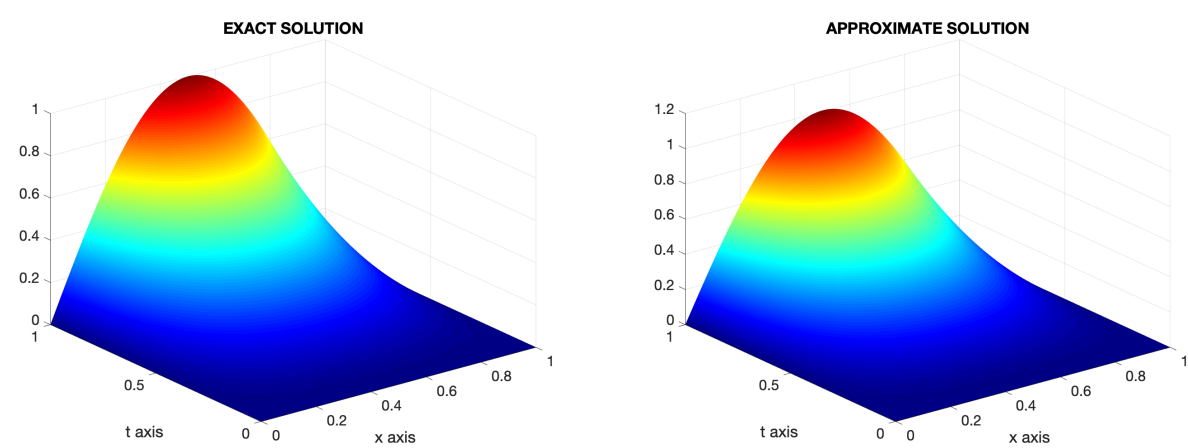

Fig. 3. Left: exact solution. Right: numerical simulation of the problem (9) for $\mathrm{N}=80, \mathrm{M}=6400$ and $\alpha=0.5$

Lastly, for $\alpha=0.99$, the exact and numerical solutions of the problem (9) are presented in Figure 4.
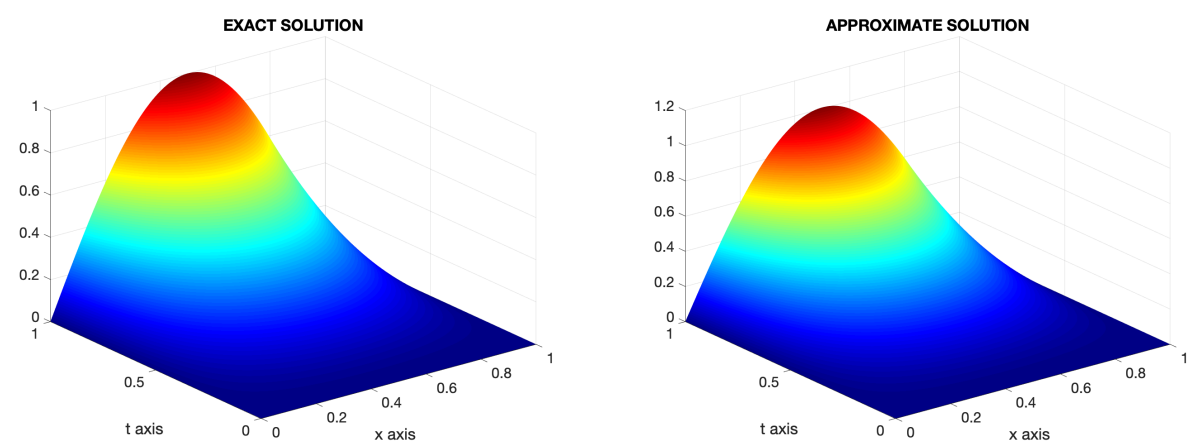

Fig. 4. Left: exact solution. Right: numerical simulation of the problem (9) for $\mathrm{N}=80, \mathrm{M}=6400$ and $\alpha=0.99$

From Figures 2-4, we conclude that numerical results are consistent with the theoretical results and the constructed difference scheme is accurate and effective for the considered problem. 

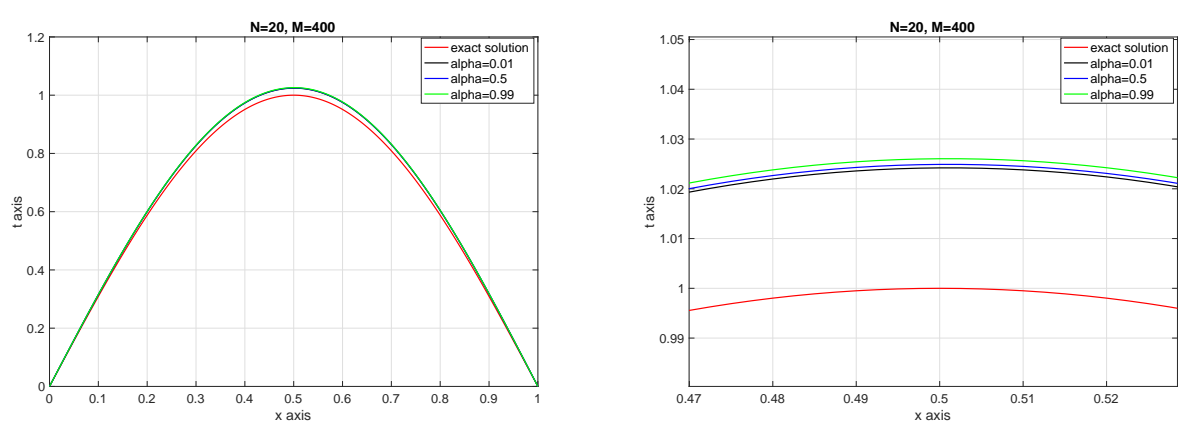

Fig. 5. Left: numerical simulations of the problem (9) for $N=20, M=400$. Right: zoom in near $(0.5,1)$ to see how, as fractional order approaches zero, the approximate and precise solutions have an almost identical match

We also compare the precise and numerical solutions of (9) in Figure 5 by varying $\alpha$ between 0 and 1 for the same grid number.

\subsection{Example 2}

We give another example to show the accuracy of the proposed method. We consider the following problem

$$
\left\{\begin{array}{l}
u_{\xi \xi}(\xi, v)+u_{\xi}(\xi, v)+u(\xi, v)+\int_{0}^{\xi} \gamma(s) D_{s}^{\alpha} u(s, v) d s=u_{\xi v v}(\xi, v)+u_{v v}(\xi, v)+f(\xi, v) \\
f(\xi, v)=\left(2+2 \xi+\xi^{2}+\frac{\xi^{2}}{\Gamma(3-\alpha)}\right)\left(v-v^{2}\right)+4 \xi+2 \xi^{2}, 0<v<1,0<\xi<1 \\
u(0, v)=u_{\xi}(0, v)=0, \quad 0 \leq v \leq 1 \\
u(\xi, 0)=u(\xi, 1)=0, \quad 0 \leq \xi \leq 1 \\
0<\alpha \leq 1
\end{array}\right.
$$

The exact solution of this problem is $u(\xi, v)=\xi^{2}\left(v-v^{2}\right)$. Then, we give the error analysis graph of the problem (12) in Figure 6 while changing time. In addition to that, we present the following error table for various values of $\alpha$ to help illustrate the error computations.

Table 2. Error analysis

\begin{tabular}{|c|c|c|c|c|c|}
\hline \multicolumn{6}{|c|}{$\tau=\frac{1}{N}, h=\frac{1}{M}, h=\tau^{2}$} \\
\hline \multicolumn{6}{|c|}{ The example (12). } \\
\hline $\mathrm{N}, \mathrm{M}$ & $\mathrm{N}=20, \mathrm{M}=20^{2}$ & $\mathrm{~N}=40, \mathrm{M}=40^{2}$ & $\mathrm{~N}=80, \mathrm{M}=80^{2}$ & $\mathrm{~N}=100, \mathrm{M}=100^{2}$ & $\mathrm{~N}=150, \mathrm{M}=150^{2}$ \\
\hline$\alpha=0.01$ & 0.0060 & 0.0032 & 0.0016 & 0.0013 & 0.0009 \\
\hline$\alpha=0.5$ & 0.0062 & 0.0033 & 0.0017 & 0.0013 & 0.0009 \\
\hline$\alpha=0.99$ & 0.0065 & 0.0034 & 0.0017 & 0.0014 & 0.0010 \\
\hline
\end{tabular}

We then present precise and approximate solutions for each $\alpha$ to demonstrate how similar the solutions are. 


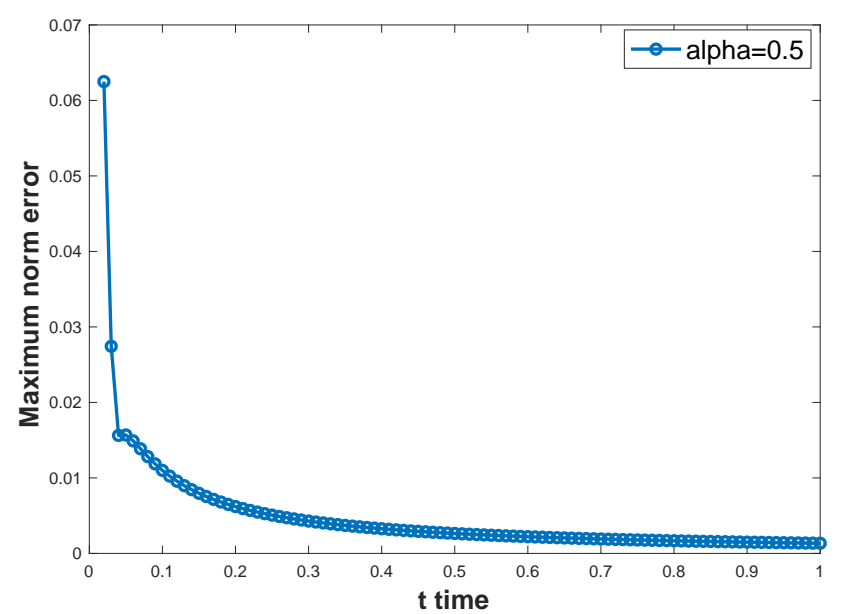

Fig. 6. Result of maximum norm errors of the difference scheme (12) for $\alpha=0.5$. As we vary the time values, it can be observed that errors keep decreasing
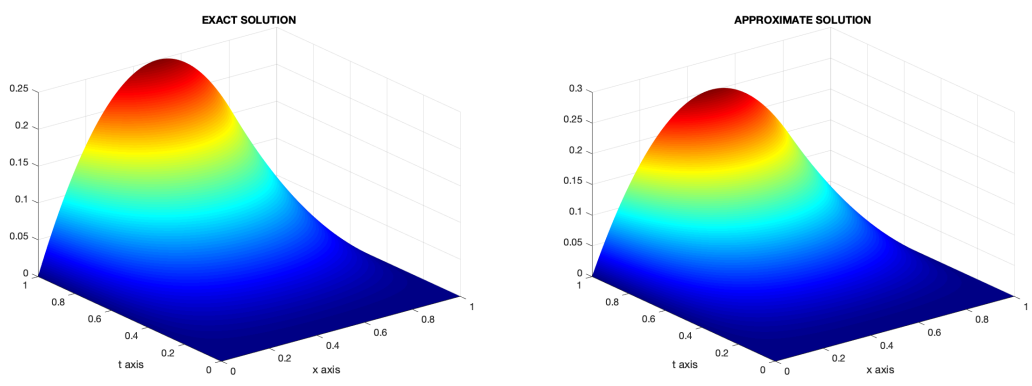

Fig. 7. Left: exact solution. Right: numerical simulation of the problem (12) for $\mathrm{N}=80, \mathrm{M}=6400$ and $\alpha=0.01$
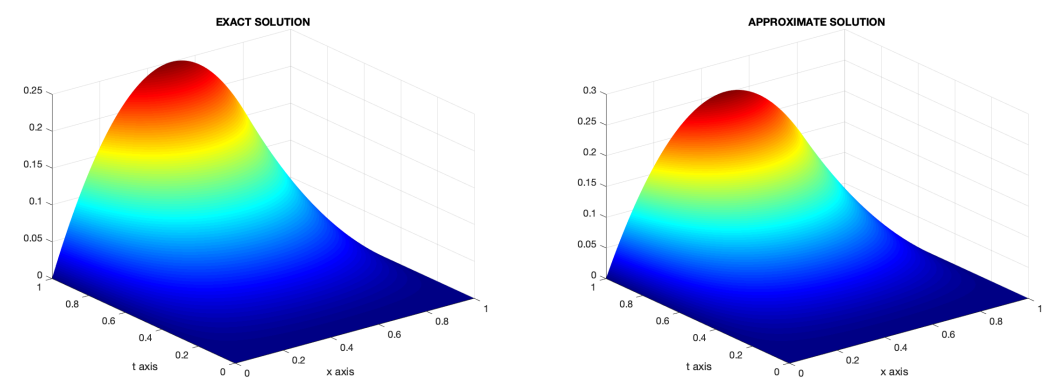

Fig. 8. Left: exact solution. Right: numerical simulation of the problem (12) for $\mathrm{N}=80, \mathrm{M}=6400$ and $\alpha=0.5$ 

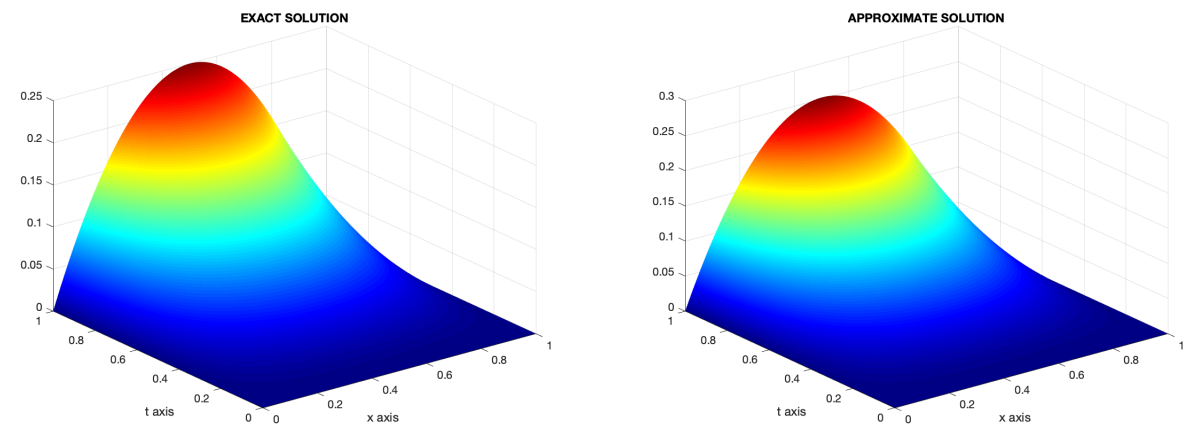

Fig. 9. Left: exact solution. Right: numerical simulation of the problem (12) for $\mathrm{N}=80, \mathrm{M}=6400$ and $\alpha=0.99$

\section{Conclusion}

In the present work, we have studied an initial-boundary value problem for the fractional order pseudo telegraph integro-differential equation. Stability estimates of the constructed difference scheme for the problem were presented.Numerical solutions for different fractional values were computed and plotted. Outputs show that the maximum norm error is decreasing while the grid points are increasing. We have provided numerical solutions of three different fractional values in order to demonstrate the efficiency and high accuracy of the difference scheme.

\section{References}

[1] Podlubny, I. (1998). Fractional Differential Equations: An Introduction to Fractional Derivatives, Fractional Differential Equations, to Methods of their Solution and Some of their Applications. Academic Press, 198.

[2] Kilbas, A.A., Srivastava, H.M., \& Trujillo, J.J. (2006). Theory and Applications of Fractional Differential Equations. 1st Edition, North-Holland Mathematical Studies.

[3] Samko, S.G., Kilbas, A.A., \& Marichev, O.I. (1993). Fractional Integrals and Derivatives: Theory and Applications. Langhorne: Gordon and Breach.

[4] Cattani, C., Srivastava, H.M., \& Yang, X.J. (2016). Fractional Dynamics. Berlin: de Gruyter.

[5] Hilfer, R. (2000). Applications of Fractional Calculus in Physics. River Edge: World Scientific Publishing.

[6] Miller, K.S., \& Ross, B. (1993). An Introduction to the Fractional Calculus and Fractional Differential Equations. 1st ed., London: Wiley.

[7] Asif, N.A., Hammouch, Z., Riaz, M.B., \& Bulut, H. (2018). Analytical solution of a Maxwell fluid with slip effects in view of the Caputo-Fabrizio derivative. The European Physical Journal Plus, 133(7), 1-13.

[8] Talib, I., Jarad, F., Mirza, M.U., Nawaz, A., \& Riaz, M.B. (2021). A generalized operational matrix of mixed partial derivative terms with applications to multi-order fractional partial differential equations. Alexandria Engineering Journal. 
[9] Ghanbari, B. (2021). A new model for investigating the transmission of infectious diseases in a prey-predator system using a non-singular fractional derivative. Mathematical Methods in the Applied Sciences.

[10] Modanli, M., \& Akgul, A. (2017). Numerical solution of fractional telegraph differential equations by theta-method. The European Physical Journal Special Topics, 229, 3693-3703.

[11] Modanli, M., \& Akgul, A. (2020). On solutions of fractional order telegraph partial differential equation by Crank-Nicholson finite difference method. Applied Mathematics and Nonlinear Sciences, 5(1), 163-170.

[12] Kumar, D., Singh, J., \& Kumar, S. (2013). Analytic and approximate solutions of space-time fractional telegraph equations via Laplace transform. Mathematics.

[13] Hosseini, V.R., Chen, W., \& Avazzadeh, Z. (2014). Numerical solution of fractional telegraph equation by using radial basis functions. Engineering Analysis with Boundary Elements, 38, 31-39.

[14] Khan, H., Shah, R., Baleanu, D., Kumam, P., \& Arif, M. (2019). Analytical solution of fractionalorder hyperbolic telegraph equation, using natural transform decomposition method. Electronics, $8(9)$.

[15] Veeresha, P., \& Prakasha, D.G. (2018). Numerical solution for fractional model of telegraph equation by using q-HATM. Mathematics.

[16] Ghanbari, B. (2020). On approximate solutions for a fractional prey-predator model involving the Atangana-Baleanu derivative. Advances in Difference Equations, 2020(1), 1-24.

[17] Nagumo, J., Arimoto, S., \& Yoshizawa, S. (1962). An active transmission line simulating nerve axon. Proceedings of the IRE, 50(10), 2061-2070.

[18] Fedotov, I., Shatalov, M.Y., \& Marais, J. (2016). Hyperbolic and pseudo-hyperbolic equations in the theory of vibration. Acta Mechanica, 227(11), 3315-3324.

[19] Chen, G., \& Yang, Z. (1993). Initial value problem for a class of nonlinear pseudo-hyperbolic equations. Acta Mathematicae Applicatae Sinica, 9(2), 166-173.

[20] Krutitskii, P.A. (1997). An initial-boundary value problem for the pseudo-hyperbolic equation of gravity-gyroscopic waves. J. Math. Kyoto Univ., 37(2), 343-365.

[21] Zhao, Z., \& Li, H. (2019). A continuous Galerkin method for pseudo-hyperbolic equations with variable coefficients. Journal of Mathematical Analysis and Applications, 473(2), 1053-1072.

[22] Riaz, M.B., Awrejcewicz, J., \& Baleanu, D. (2021). Exact Solutions for Thermomagetized Unsteady Non-singularized Jeffrey Fluid: Effects of Ramped Velocity, Concentration with Newtonian Heating. Results in Physics, 104367.

[23] Ghanbari, B. (2021). On novel nondifferentiable exact solutions to local fractional Gardner's equation using an effective technique. Mathematical Methods in the Applied Sciences, 44(6), 4673-4685.

[24] Au, V.V., Jafari, H., Hammouch, Z., \& Tuan, N.H. (2021). On a final value problem for a nonlinear fractional pseudo-parabolic equation. Electronic Research Archive, 29(1), 1709-1734. doi: 10.3934/era.2020088.

[25] Can, N.H., Kumar, D., Viet, T.V., \& Nguyen, A.T. (2021). On time fractional pseudo-parabolic equations with nonlocal integral conditions. Mathematical Methods in the Applied Sciences. https://doi.org/10.1002/mma.7196.

[26] Inc, M., Partohaghighi, M., Akinlar, M.A., Agarwal, P., \& Chu, Y.M. (2020). New solutions of fractional-order Burger-Huxley equation. Results in Physics, 18, 103290.

[27] Partohaghighi, M., Bayram, M., \& Baleanu, D. (2019). On numerical solution of the time fractional advection-diffusion equation involving Atangana-Baleanu-Caputo derivative. Open Physics, 17(1), 816-822. 
[28] Parto-Haghighi, M., \& Manafian, J. (2020). Solving a class of boundary value problems and fractional Boussinesq-like equation with $\beta$-derivatives by fractional-order exponential trial functions. Journal of Ocean Engineering and Science, 5(3), 197-204.

[29] Inc, M., Parto-Haghighi, M., Akinlar, M.A., \& Chu, Y.M. (2021). New numerical solutions of fractional-order Korteweg-de Vries equation. Results in Physics, 19, 103326.

[30] Lundgren, L., \& Mattsson, K. (2020). An efficient finite difference method for the shallow water equations. Journal of Computational Physics, 422(1), 109784.

[31] Li, P.W. (2020). Space-time generalized finite difference nonlinear model for solving unsteady Burgers equations. Applied Mathematics Letters, 114, 106896.

[32] Modanli, M. (2019). On the numerical solution for third order fractional partial differential equation by difference scheme method. An International Journal of Optimization and Control: Theories and Applications (IJOCTA), 9(3), 1-5. 